\title{
Nuclear Role of LOX-1 in XinMaiJia-attenuating Atherosclerosis in Rabbits
}

\author{
R. L. SUN, T. T. ZHU1 , M. L. ZHU1 , P. SONG ${ }^{1}$, J. XU1 , Y. L. YIN²* AND P. LI ${ }^{1 *}$
}

Henan Key Laboratory of Immunology and Targeted Therapy, Henan Collaborative Innovation Center of Molecular Diagnosis and Laboratory Medicine, School of Laboratory Medicine, ${ }^{1}$ College of Pharmacy, ${ }^{2}$ School of Basic Medical Sciences, Xinxiang Medical University, Xinxiang, Henan 453003, China

\section{Sun et al.: Nuclear role of LOX-1 in XinMaiJia-attenuating atherosclerosis}

\begin{abstract}
This study aimed to explore the nuclear role of lectin-like oxidised low density lipoprotein receptor-1 in atherosclerotic rabbits treated with XinMaiJia to further confirm that low density lipoprotein receptor-1 is a target of XinMaiJia in attenuating atherosclerosis. Atherosclerotic model of rabbits was established. Lovastatin, zhibituo and different concentrations of XinMaiJia were administered to atherosclerotic rabbits. Pathological examination was performed on the right common carotid artery, cytokines reflected inflammation and oxidative stress level were detected. Moreover, endothelin-1, low density lipoprotein receptor-1 and endothelial nitric oxide synthase expression were measured. XinMaiJia prevented the development of atherosclerosis and reduced low density lipoprotein receptor-1 expression in rabbits. For antioxidant effect, XinMaiJia increased superoxide dismutase activity but decreased malondialdehyde level in plasma. For antiinflammation effect, XinMaiJia increased the expression of endothelial nitric oxide synthase and nitric oxide, but reduced intercellular adhesion molecule-1, vascular cell adhesion molecule-1, matrix metalloprotein-2 and nuclear factor-kappa B levels. Furthermore, XinMaiJia reduced endothelin-1 expression. More importantly, all these changes have a feedback with low density lipoprotein receptor-1. XinMaiJia increased the antioxidant and antiinflammation capacity of the body to prevent the development of atherosclerosis. The possible mechanisms of XinMaiJia were mainly through inhibiting the vital gene low density lipoprotein receptor-1.
\end{abstract}

Key words: XinMaiJia, lectin-like oxidised low density lipoprotein receptor-1, inflammation, oxidant, endothelin-1

Atherosclerosis (AS) is currently one of the severest diseases in humans ${ }^{[1]}$. However, the mechanisms of this disease are complicated. Hyperlipidaemia is the most important risk factor of $\mathrm{AS}^{[2]}$. Low density lipoprotein is oxidised to oxidised low density lipoprotein (OLDL), which induces AS. Studies show that OLDL has some biologic characteristics, such as causing vascular endothelial cells (ECs) injury. This lipoprotein also promotes platelet aggregation, adhesion and thrombosis. Moreover, phagocytes distinguish OLDL, thereby leading to cholesterol ester aggregation and resulting in the formation of foam cells, which are features of early atherosclerotic lesions. Immunologic stain analysis proves that OLDL locates in the atherosclerotic plaque, especially in the unstable atherosclerotic plaque ${ }^{[3]}$. This finding indicates that only OLDL could promote AS, and inhibiting OLDL delays $\mathrm{AS}^{[4,5]}$.

Lectin-like oxidised low density lipoprotein receptor-1 (LOX-1), the major receptor of OLDL in endothelial

*Address for correspondence E-mail: pengli@xxmu.edu.cn

November-December 2019 cells, is specifically bound to OLDL ${ }^{[6]}$. LOX-1 induces ECs injury by mediating ECs uptake OLDL, thereby promoting foam cells formation and smooth muscle cells proliferation, and eventually accelerates AS. Many studies ${ }^{[7-9]}$ have found that the expression of LOX-1 increases in both ECs and macrophages of atherosclerotic plaque. Furthermore, LDL geneknock-out mice could delay the development of AS and soluble LOX-1 is a predictable factor of AS. The above evidences demonstrate that the interaction of LOX-1 and OLDL plays a key role in AS and LOX-1 is probable a new target in the treatment of AS.

This is an open access article distributed under the terms of the Creative Commons Attribution-NonCommercial-ShareAlike 3.0 License, which allows others to remix, tweak, and build upon the work non-commercially, as long as the author is credited and the new creations are licensed under the identical terms

Accepted 21 September 2019

Revised 14 June 2019

Received 02 March 2019

Indian J Pharm Sci 2019;81(6):1020-1028 
XinMaiJia (XMJ), a Chinese medicinal formulation, which is available in capsule form. XMJ contains functional red kojic rice powder (10-35\%), kudzu flavonoid powder (1-10 \%), soybean isoflavone powder (1-8 \%), bamboo leaf flavone powder (1-8\%), resveratrol powder (1-8\%), hawthorn powder (1-6\%), gastrodia powder (1-6\%), Auricularia auricula powder (1-30\%), hippocampus powder (0.1-0.2\%), astaxanthin powder (0.008-0.04\%), menthol powder (0.1-0.3\%) and resistant starch (20-50\%). XMJ has antiinflammation and antioxidant effects, thus significantly inhibiting the proliferation and migration of human aortic smooth muscle cells ${ }^{[10]}$. A previous study has shown that XMJ could alleviate cardio- and cerebrovascular diseases and reduce blood lipids ${ }^{[11,12]}$. LOX-1 is associated with inflammation and oxidative stress in AS. However, whether the antiinflammation and antioxidant effects of XMJ are through LOX-1 has not been confirmed.

Rabbits with AS were established and used in the present study. The expression of LOX-1, inflammatory cytokines levels and oxidative indices were detected to reflect inflammatory response and the level of oxidation. Furthermore, this study would provide experimental evidences for drug research and development through analysis of possible mechanisms, which associated with LOX-1 to relieve AS.

\section{MATERIALS AND METHODS}

Forty-eight Japanese white rabbits were obtained from Henan Kangda Laboratory Animal Co., Ltd. All animals received humane care in accordance with the National Institutes of Health Guide for the Care and Use of Laboratory Animals (National Institutes of Health, 2011). This animal research was approved by the Institutional Animal Care and Use Committee (IACUC) of Henan Xinxiang Medical University.

Lovastatin used in these animal experiments was purchased from Sigma Company. Zhibituo was purchased from DiaoJiuhong Pharmaceutical Industry. $\mathrm{XMJ}$ was composed of astaxanthin, functional red rice, pueraria isoflavone, soybean isoflavone, bamboo leaf flavones and resveratrol, which were purchased from Beijing Tongrentang Co., Ltd.

\section{Animal experimental protocols:}

Forty-eight Japanese white rabbits were maintained at $25^{\circ}$ under a 12-h light/12-h dark cycle. After $1 \mathrm{w}$ of adaptation period, animals were randomly segregated into 8 groups ( $n=6$ per group) and the rabbits were fed with a conventional diet in the normal group. The rabbits were fed with a conventional diet and intragastric (ig) administration of $0.69 \mathrm{~g} / \mathrm{kg} / \mathrm{d}$ sodium citrate for the last $10 \mathrm{w}$ in the vehicle control group. The rabbits were fed with a high-fat diet for $4 \mathrm{w}$, injected vitamin D3 (300 $000 \mathrm{U} / 200 \mathrm{mg}$ ), and were induced balloon injury to develop diabetes ${ }^{[11-16]}$ in the model group, then the rabbits were fed with a high-fat diet for another $10 \mathrm{w}$. In the lovastatin group, diabetes rats were fed with a high-fat diet and intragastric administration of $2.4 \mathrm{mg} / \mathrm{kg} / \mathrm{d}$ lovastatin for the last $10 \mathrm{w}$. In the zhibituo group, diabetes rats were fed with a highfat diet along with intragastric administration of $0.3125 \mathrm{~g} / \mathrm{kg} / \mathrm{d}$ zhibituo for the last $10 \mathrm{w}$. In the XMJ (L) group, diabetes rats were fed with a high-fat diet and intragastric administration of $0.2184 \mathrm{~g} / \mathrm{kg} / \mathrm{d}$ XMJ for the last $10 \mathrm{w}$. In XMJ (M) group, diabetes rats were fed with a high-fat diet and intragastric administration of $0.69 \mathrm{~g} / \mathrm{kg} / \mathrm{d}$ XMJ for the last $10 \mathrm{w}$. In XMJ (H) group, diabetes rats were fed with a high-fat diet and intragastric administration of $2.1804 \mathrm{~g} / \mathrm{kg} / \mathrm{d}$ XMJ for the last $10 \mathrm{w}$.

The high-fat diet was composed of basic diet (81.5\%), lard $(10 \%)$, sodium cholate $(0.5 \%)$, cholesterol (3\%) and sugar $(5 \%)$ was given to all the rabbits except for the normal control and vehicle control group during the entire treatment period. The dose of the high-fat diet was $150 \mathrm{~g} / \mathrm{d}$. Common carotid arterial intima injury was performed after the rabbits fed for $4 \mathrm{w}$.

\section{Histological measurements:}

After the rabbits were anaesthetised, $1.5 \mathrm{~cm}$ long common carotid arteries were obtained and firmed. The samples were made into paraffin sections (SLEE automatic freezing microtome, Germany) through dehydration, transparency, wax dip and embedment. Sections were stained with hematoxylin-eosin (HE) and detected by immunohistochemistry assay. The sections were then observed under an optic microscope $(\times 400)$.

\section{ELISA assay:}

Approximately $2 \mathrm{ml}$ of blood was extracted from the common carotid artery. The extracted blood was placed into a tube to mix equably. Supernatant was obtained after centrifugation at a speed of $3000 \mathrm{rpm}$ at $4^{\circ}$ for 15 min (SLEE automatic freezing microtome, Germany). Following the manufacturer's instructions of ELISA kit (Sigma Company, USA), blood samples 
were analysed to detect related inflammatory factor and oxidative index.

\section{Immunohistochemistry assay:}

Sections were dewaxed and then incubated in $3 \%$ $\mathrm{H}_{2} \mathrm{O}_{2}$ for $5 \mathrm{~min}$ to $10 \mathrm{~min}$ at room temperature. Subsequently, sections were incubated at 92 to $98^{\circ}$ for $30 \mathrm{~min}$. Afterwards, primary antibodies, including LOX-1, eNOS and ET-1 (1:100 dilutions) were added, and negative control was prepared with PBS instead of primary antibody. All samples were placed in a refrigerator overnight at $4^{\circ}$. The samples were washed and incubated for $1 \mathrm{~h}$ with horseradish peroxidaseconjugated secondary antibodies. After incubation at $37^{\circ}$ for $30 \mathrm{~min}$, samples were stained with DAB and restained with haematoxylin. The samples were then dehydrated and slices were prepared. All kits were purchased from Sigma Company (USA).

\section{Western blot:}

Protein was extracted from the aorta then lysed with cold protein lysis buffer containing $150 \mathrm{mM} \mathrm{NaCl}$, $50 \mathrm{mM}$ Tris-HCl, pH 7.4, $10 \mathrm{mM}$ EDTA pH 8.0, $1 \%$ Triton X-100, $1 \%$ deoxycholate, $0.1 \%$ sodium dodecyl sulphate (SDS), $0.1 \%$ protease inhibitors and $0.1 \%$ PMSF. The lysates were centrifuged at $12000 \mathrm{rpm}$ for $15 \mathrm{~min}$ at $4^{\circ}$ and the protein concentrations were determined by BCA protein assay kit (Beyotime, China). A fixed amount of protein (20-60 $\mu \mathrm{g})$ was separated from each sample by using $10 \% \mathrm{SDS} /$ polyacrylamide gel electrophoresis (SDS/PAGE). These proteins were transferred onto polyvinylidenefluoride (PVDF) membranes (Millipore, USA). Then the membranes were blocked with $5 \%$ skimmed milk powder for 90 min and incubated the LOX-1 (1:1000; Abcam, Cambridge, MA, USA) at $4^{\circ}$ for one night. Finally the membrane was incubated with a peroxidaseconjugated mouse antirabbit antibody (1:5000; Santa Cruz, CA, USA) at room temperature for $1 \mathrm{~h}$. The chemiluminescence signals were detected using Easy See Western Blot Kit (Beijing TransGen Biotech, Beijing, China). Densitometric analysis was conducted using Image J 1.43 software (National Institutes of Health, New York City, NY, USA).

\section{Statistical analysis:}

SPSS software (Version 18) was used for statistical analysis. Data were expressed as mean \pm SD. Statistical analysis was performed by unpaired Student's t-test for two groups; one-way ANOVA followed by Newman-
Student-Keuls test was performed for multiple groups. Differences were considered statistically significant when $\mathrm{p}<0.05$.

\section{RESULTS AND DISCUSSION}

XMJ prevented the development of AS in rabbits, when compared with the control group; endothelium injury and yellow plaque were obvious in the model group. $\mathrm{XMJ}$ could alleviate vascular endothelium injury and minimise or diminish plaque in a dose-dependent manner. Lovastatin also alleviated the vascular endothelium injury in rabbits and the plaque was smaller in the lovastatin group. However, lovastatin had some effects on endothelial continuity. In addition, zhibituo does not have a significant effect (fig. 1A).

Under an optical microscope, it was observed that $\mathrm{XMJ}$ in a dose-dependent manner made the vascular endothelium smooth in rabbits with AS. The nuclei of ECs were stained uniformly. The gaps between cells were relatively distinct, and the muscular layers under the endothelia were arranged regularly (fig. 1B). And

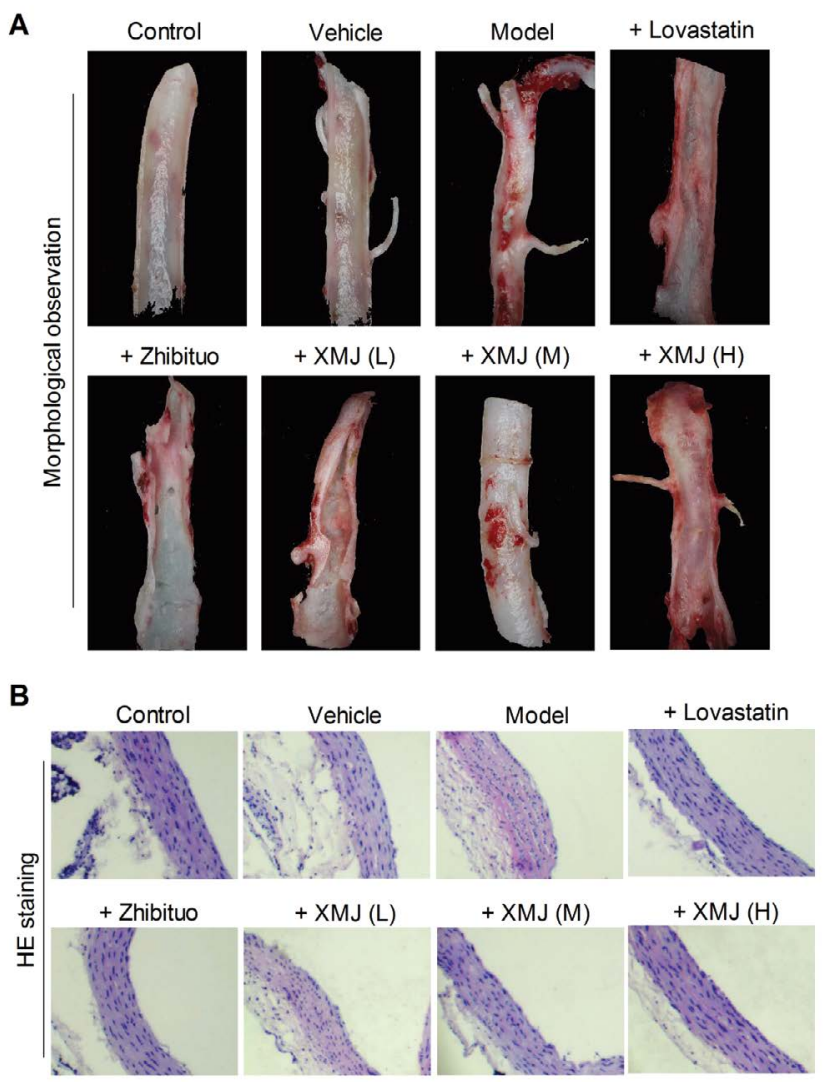

Fig. 1: XMJ prevented the development of atherosclerosis in rabbits

(A) morphology observation of carotid artery with the naked eye, (B) HE staining of carotid artery. Magnification, $\times 400$. $\mathrm{n}=6$. XMJ (L), low dose of XinMaiJia; XMJ (M), medial dose of XinMaiJia; XMJ (H), high dose of XinMaiJia; HE staining, haematoxylin and eosin staining 
the high dose XMJ group showed the best effects among the treatment groups.

Fig. 2 showed that compared with the control group, the expression of LOX-1 was significantly increased in the model group $(p<0.05)$. Furthermore, the LOX-1 expression was lower with dose dependence in the XMJ groups and zhibituo group than that in the model group $(p<0.05)$. Moreover, compared with other groups, the expression of LOX-1 reduced significantly in the high dose XMJ group.

XMJ significantly reduced the level of intercellular adhesion molecule-1 (ICAM-1), vascular cell adhesion molecule-1 (VCAM-1), matrix metalloprotein-2 (MMP-2) and nuclear factor-kappa B (NF-kB), but increased the expression of $\mathrm{NO}$ in a dose-dependent manner in AS rabbits. A significant difference

A

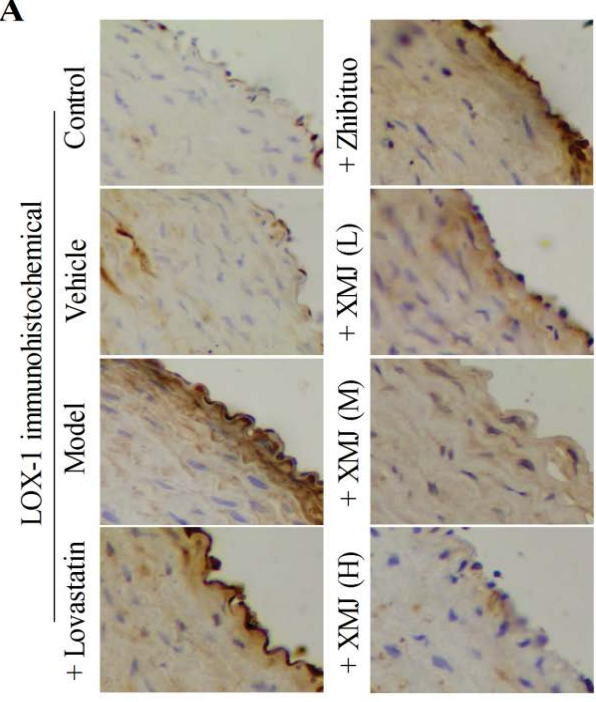

exists between XMJ and model groups $(\mathrm{p}<0.05$, Table 1). Moreover, compared with other groups, the inflammatory response reduced significantly in the high dose XMJ group.

Fig. 3 showed that compared with the control group, the eNOS expression was increased in the model group $(p<0.05)$; the eNOS expression was higher in the XMJ groups than those in the model group $(p<0.05)$. Moreover, compared with other groups, the eNOS expression increased significantly in the high dose $\mathrm{XMJ}$ group.

XMJ inhibited endothelial oxidative stress via controlling oxidative indexes. Superoxide dismutase (SOD) and malondialdehyde (MDA) level were detected to evaluate the antioxidation effect of XMJ. Current results showed that compared with the control

\section{B}

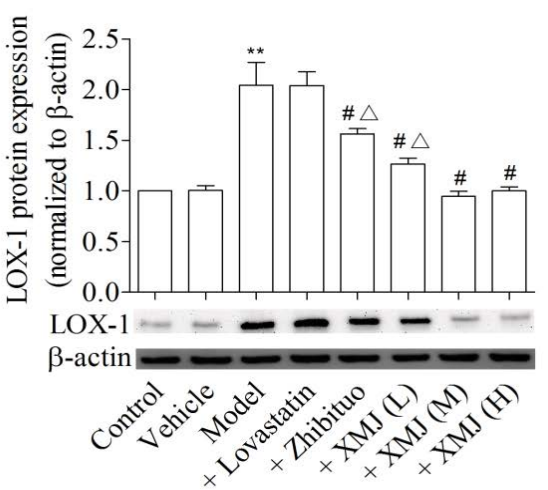

Fig. 2: XMJ alleviated endothelial injury via inhibiting the expression of LOX-1

(A) immunohistochemical staining of endothelial LOX-1 in the vascular tissue. Magnification, $\times 400$; (B) LOX-1 protein expression of the vascular tissue. XMJ (L), low dose of XinMaiJia; XMJ (M), medial dose of XinMaiJia; XMJ (H), high dose of XinMaiJia; LOX-1, lectin-like oxidized low-density lipoprotein receptor-1. Data are shown as mean \pm SD. $n=6,{ }^{*} p<0.05$, vs. control, ${ }^{\# p}<0.05$, vs. model; ${ }^{\Delta} \mathbf{p}<0.05$, vs. + XMJ (H)

\section{TABLE 1: RELATED CYTOKINES LEVELS OF JAPANESE WHITE RABBITS IN PLASMA}

\begin{tabular}{|c|c|c|c|c|c|}
\hline Group & ICAM-1 (nM) & VCAM-1 $(\mu M)$ & MMP-2 $(\mu M)$ & NF-kB (nM) & $\mathrm{NO}(\mu \mathrm{M})$ \\
\hline Control & $322.39 \pm 42.66$ & $29.57 \pm 3.38$ & $6.27 \pm 0.54$ & $598.99 \pm 45.55$ & $29.87 \pm 3.76$ \\
\hline Vehicle & $368.47 \pm 32.01$ & $30.27 \pm 4.52$ & $6.33 \pm 0.61$ & $568.25 \pm 56.79$ & $28.66 \pm 2.65$ \\
\hline Model & $987.45 \pm 54.12^{*}$ & $55.41 \pm 6.34^{*}$ & $9.97 \pm 0.87^{*}$ & $2257.39 \pm 124.47^{*}$ & $12.32 \pm 1.27^{*}$ \\
\hline +Lovastatin & $745.25 \pm 68.47^{* \# \Delta}$ & $48.44 \pm 5.27^{* \# \Delta}$ & $7.24 \pm 0.65^{* \# \Delta}$ & $1458.43 \pm 92.54^{*} \# \Delta$ & $19.33 \pm 1.47^{* \# \Delta}$ \\
\hline +Zhibituo & $687.42 \pm 66.31^{* \# \Delta}$ & $45.33 \pm 4.87^{* \# \Delta}$ & $7.22 \pm 0.75^{* \# \Delta}$ & $1368.42 \pm 78.54^{* \# \Delta}$ & $18.86 \pm 1.63^{* \# \triangle}$ \\
\hline +XMJ (L) & $654.74 \pm 62.19^{* \# \Delta}$ & $42.39 \pm 4.28^{* \# \Delta}$ & $7.54 \pm 0.37^{* \# \Delta}$ & $1212.23 \pm 65.09^{* \# \Delta}$ & $19.85 \pm 1.63^{* \# \triangle}$ \\
\hline$+\mathrm{XMJ}(\mathrm{M})$ & $487.93 \pm 54.23^{* \# \Delta}$ & $35.47 \pm 3.68^{* \# \Delta}$ & $6.87 \pm 0.69^{* \# \Delta}$ & $899.74 \pm 75.52^{* \# \Delta}$ & $22.39 \pm 1.47^{* \# \triangle}$ \\
\hline$+\mathrm{XMJ}(\mathrm{H})$ & $469.38 \pm 43.66^{* \#}$ & $36.57 \pm 4.31^{* \#}$ & $6.75 \pm 0.65^{* \#}$ & $824.37 \pm 87.53^{* \#}$ & $19.76 \pm 2.03^{* \#}$ \\
\hline
\end{tabular}

ICAM-1, VCAM-1, MMP-2, NF-KB and NO levels of Japanese white rabbits in plasma. XMJ (L), low dose of XinMaiJia; XMJ (M), medial dose of XinMaiJia; XMJ $(\mathrm{H})$, high dose of XinMaiJia; ICAM-1, intercellular adhesion molecule-1; VCAM-1, vascular cell adhesion molecule-1; MMP-2, matrix metalloprotein-2; NF-kB, nuclear factor-kappa B; NO, nitric oxide. Data are shown as mean $\pm S D$. $n=6$, " $p<0.05$, vs. control, " $p<0.05$, vs. model; ${ }^{\wedge} \mathrm{p}<0.05$, vs. $+\mathrm{XMJ}(\mathrm{H})$ 
group, SOD level was obviously reduced, but the MDA level was increased in the model group. Furthermore, compared with the model group, the SOD level were significantly increased, but the MDA level was reduced in the lovastatin, zhibituo and XMJ groups with different concentrations $(\mathrm{p}<0.05)$. Compared with other groups, the SOD level increased, but the MDA level decreased in the high-dose XMJ group (Table 2). These results indicated that XMJ could dramatically increase the NO and SOD level and decline the MDA level in AS rabbits. A significant difference existed between XMJ and model groups $(\mathrm{p}<0.05)$.

$\mathrm{XMJ}$ protected endothelial function via inhibiting ET-1 in the vascular tissue. Fig. 4 showed that compared with the control group, the expression of ET-1 was significantly increased in model group $(p<0.05)$. Furthermore, ET-1 expression was lower with dosedependence in the XMJ groups than those in model group $(\mathrm{p}<0.05)$. Compared with other groups, the ET-1

TABLE 2: OXIDATION INDEXES OF JAPANESE WHITE RABBITS IN PLASMA

\begin{tabular}{lcc}
\hline Group & SOD $(\mathrm{kU} / \mathrm{l})$ & MDA $(\mu \mathrm{mol} / \mathrm{l})$ \\
\hline Control & $44.54 \pm 5.45$ & $12.65 \pm 1.22$ \\
Vehicle & $46.67 \pm 4.36$ & $13.87 \pm 1.65$ \\
Model & $19.36 \pm 1.98^{*}$ & $42.58 \pm 3.58^{*}$ \\
+Lovastatin & $23.69 \pm 2.36^{* \# \Delta}$ & $33.94 \pm 3.57^{* \# \Delta}$ \\
+Zhibituo & $32.58 \pm 3.22^{* \# \Delta}$ & $32.59 \pm 3.27^{* \# \Delta}$ \\
+XMJ (L) & $26.55 \pm 3.24^{* \# \Delta}$ & $33.27 \pm 3.53^{* \# \Delta}$ \\
+XMJ (M) & $39.87 \pm 3.66^{* \# \Delta}$ & $36.52 \pm 2.89^{* \# \Delta}$ \\
+ XMJ (H) & $38.42 \pm 3.75^{* \#}$ & $35.45 \pm 4.44^{* \#}$ \\
\hline
\end{tabular}

SOD and MDA levels of Japanese white rabbits in plasma. XMJ (L), low dose of XinMaiJia; XMJ (M), medial dose of XinMaiJia; XMJ $(\mathrm{H})$, high dose of XinMaiJia; SOD, superoxide dismutase; MDA, malonaldehyde. Data are shown as mean $\pm S D$. $n=6, " p<0.05$, vs. control, ${ }^{*} \mathrm{p}<0.05$, vs. model; ${ }^{\Delta} \mathrm{p}<0.05$, vs. $+\mathrm{XMJ}(\mathrm{H})$ expression decreased significantly in the high dose $\mathrm{XMJ}$ group.

ECs injury in vascular vessels is critically involved in the formation and development of $\mathrm{AS}^{[17]}$. XMJ, a Chinese medicinal formulation, which has antiinflammation and antioxidant effects, thus significantly inhibiting the proliferation and migration of human aortic smooth muscle cells ${ }^{[10]}$. However, the mechanisms behind the antiinflammation and antioxidant effects of XMJ on AS in ECs have not been clearly defined. Our data presented here was the first to show the effectiveness of $\mathrm{XMJ}$ in inhibiting ECs injury via suppressing LOX-1 expression. The data suggested that XMJ inhibited inflammation and oxidative stress level via reversing the upregulation of LOX-1 in ECs in vivo (fig. 2). Furthermore, it is suggested that XMJ may potentially be used as a medicinal compound for the treatment of AS via suppressing LOX-1 expression.

OLDL, which is the ligand of LOX-1, could trigger the oxidation of blood vessels especially the reactive oxygen species such as superoxide anion radical and could injure the ECs. Superoxide anion radical is oxygen free radical. The oxygen free radical could not only oxidise and destruct the vascular ECs but also oxidise LDL and form OLDL. Then OLDL could induce the ECs to express ICAM-1 and VCAM- ${ }^{[18,19]}$. ICAM-1 and VCAM-1 promote the infiltration of immunocytes, making monocytes and lymphocytes to adhere and migrate through the endothelium. The monocytes would then differentiate into macrophages, expressing the scavenger receptor that could recognise OLDL. The cholesterol deposit would lose the limitation, thus contributing to the formation of foam

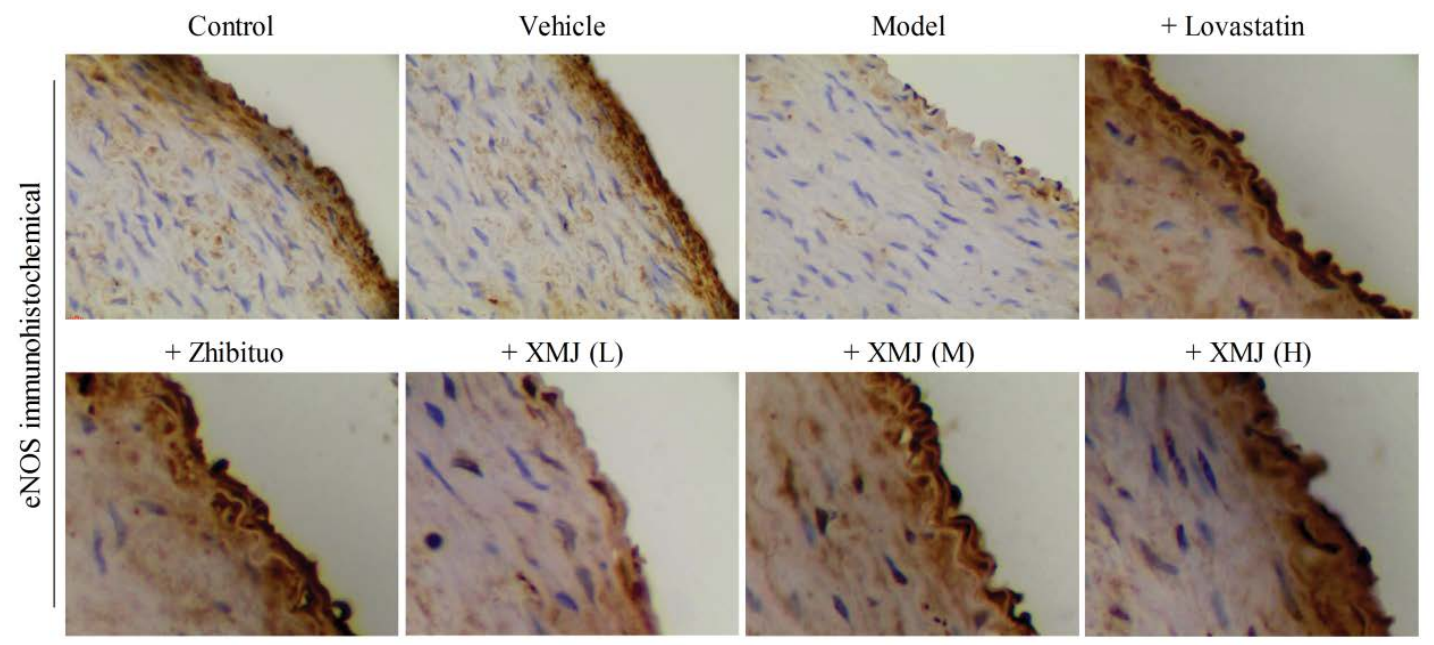

Fig. 3: XMJ inhibited endothelial inflammation via promoting the expression of eNOS Immunohistochemical staining of eNOS in the vascular tissue. XMJ (L), low dose of XinMaiJia; XMJ (M), medial dose of XinMaiJia; XMJ (H), high dose of XinMaiJia; eNOS: endothelial nitric oxide synthase. $n=6$, magnification, $\times 400$ 


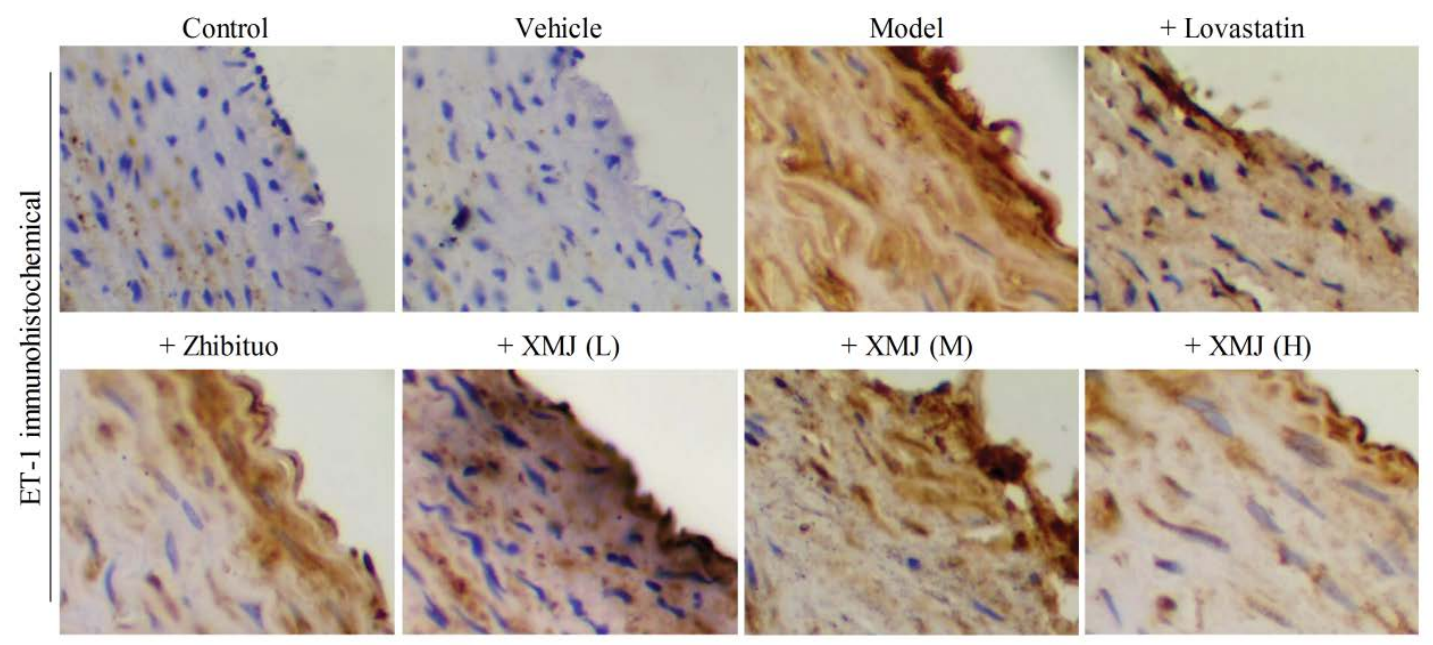

Fig. 4: XMJ protected endothelial function via inhibiting ET-1 in the vascular tissue Immunohistochemical staining of ET-1 in the vascular tissue. XMJ (L), low dose of XinMaiJia; XMJ (M), medial dose of XinMaiJia; XMJ (H), high dose of XinMaiJia; ET-1: endothelin. n=6, magnification, $\times 400$

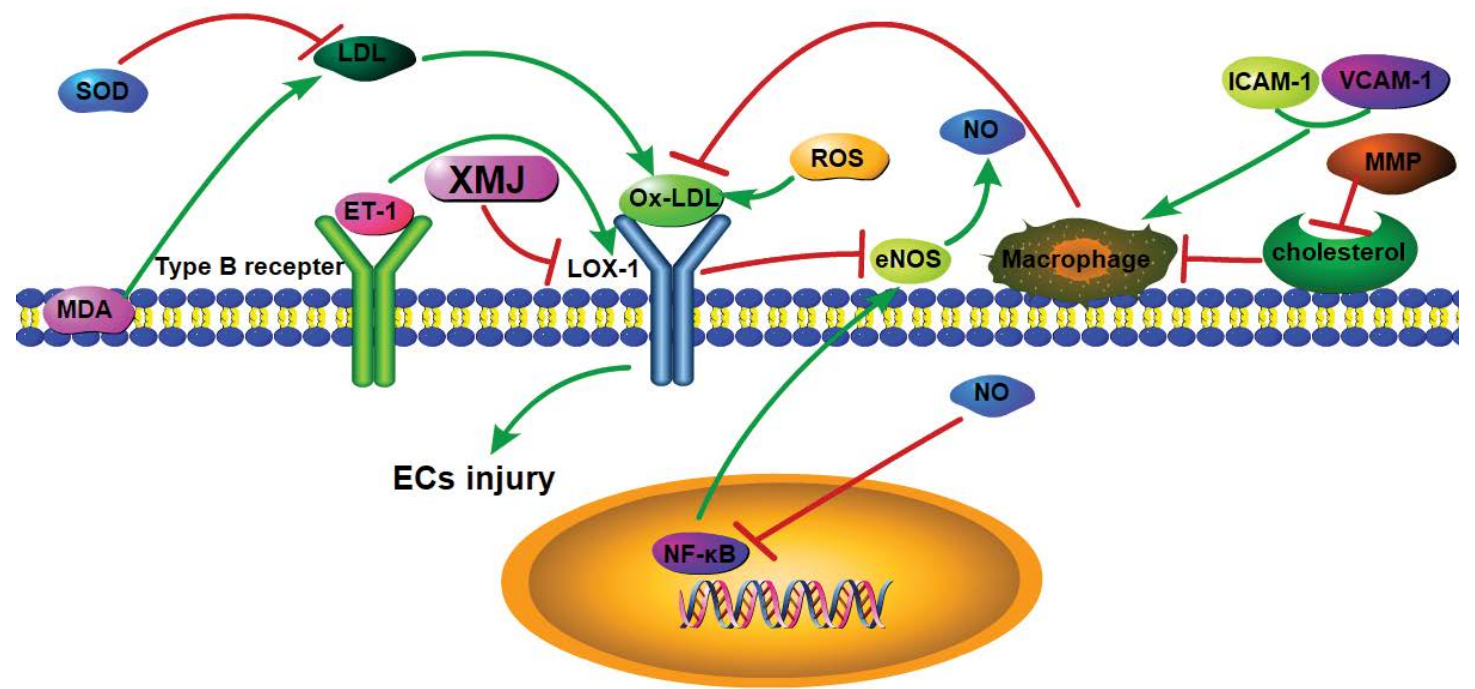

Fig. 5: The possible mechanisms of XMJ protecting endothelial function

Green arrows show promoting, red arrows show inhibiting. XMJ, XinMaiJia; LOX-1, lectin-like oxidized low-density lipoprotein receptor-1; ET-1: endothelin; eNOS: endothelial nitric oxide synthase; SOD, superoxide dismutase; MDA, malonaldehyde; ICAM1, intercellular adhesion molecule-1; VCAM-1, vascular cell adhesion molecule-1; MMP-2, matrix metalloprotein-2; NF-кB, nuclear factor-kappa B; NO, nitric oxide

cells and the occurrence of $\mathrm{AS}^{[20]}$. In present study, it was found that the serum inflammation-associated cytokines in model group, such as ICAM-1, VCAM-1, $\mathrm{NF}-\kappa \mathrm{B}$ and MMP were significantly increased. These results indicated that during hyperlipidaemia, the body triggered an inflammatory response, which promoted the occurrence of AS. After administration of XMJ, the inflammation-associated cytokines were significantly reduced, thereby generating an antiAS effect. Notably, the decrease in extracellular matrix synthesis and the increase in degradation are the main reasons towards the rupture of AS plaques. MMP-2 is also an important factor towards the degradation of extracellular matrix ${ }^{[21-23]}$. This study found that the plasma MMP-2 in the model group was significantly higher. After administration of XMJ, the MMP-2 level was significantly reduced, indicating that XMJ might help stabilize the AS plaques by reducing the expression of MMP-2.

MDA is a lipid peroxidised metabolite generated by the free radicals-reacted polyunsaturated fatty acids within the biofilm. The MDA content could reflect the rate and extent of lipid peroxidation, representing the activities of free radicals. Moreover, MDA could promote LDL per-oxidation and damage endothelial cells. SOD 
is the in vivo synthesised scavenger that towards the oxygen-free radicals. The SOD activity could reflect the body's ability to scavenge free radicals, prevent excessive LDL oxidation and protect the ECs. The results of our experiment revealed that the serum SOD level of the model group was significantly reduced. By contrast, the MDA level was significantly increased. This finding indicated that during hyperlipidaemia, the body's antioxidation ability reduced, the oxygen radicals were largely generated and the oxidative injuries were obvious, thus promoting the formation of AS. After administration of XMJ, the activity of SOD was significantly increased, the free radical injuries and the content of MDA were reduced. The injury of vascular endothelium caused by the lipid peroxidation was reduced.

ECs could uptake OLDL through its specific receptor LOX-1. This phenomenon activates NADPH oxidase to produce large amounts of ROS, promotes the activation of $\mathrm{NF}-\kappa \mathrm{B}$, inducing oxidative and inflammatory effects ${ }^{[24,25]}$. Studies have shown that the increased expression of LOX-1 is related to AS, and drugs that inhibit the expression of LOX-1 could prevent the early stage of $\mathrm{AS}^{[26-30]}$. In our experiment we found that the expression of LOX-1 in model group was significantly increased. This finding indicated that during hyperlipidaemia, the body initiated an oxidative and inflammatory response, which promoted AS. After administration of XMJ, LOX-1 was significantly decreased, thereby reducing the oxidative and inflammatory effects.

Endothelin (ET) is a peptide composed by 21 amino acids. ET was isolated from the porcine aortic ECs by Yanagisiawa in 1988. Many tissues in vivo could synthesise ET. The ECs could produce ET-1, exhibiting the strong and lasting vasoconstriction effect in vitro and in vivo. Increased ET level is a sign of endothelial dysfunction and is involved in the occurrence and development of AS. The in vitro experiment found that ET-1 binds its type $B$ receptor to promote the expression of LOX-1 mRNA and protein, thus promoting the uptake of OLDL and contributing to the endothelial dysfunction. The ET blocker may block this effect ${ }^{[31]}$. Sakurai found that LOX-1 could combine CD40 and promote the expression of ET-1 on the platelet surface to promote platelet adhesion ${ }^{[31]}$. These findings suggested that ET-1 and LOX-1 could mutually promote and cowork in AS.
$\mathrm{NO}$ is a type of endothelium-derived relaxing factor and is catalytically synthesised by eNOS, which was on the surface of vascular endothelial cells. Under physiological conditions, it has blood vessel-protecting activities, such as vessel dilation, antiplatelet aggregation and antiproliferation of vascular smooth muscle cells. Once the inflammatory cells enter the vessel wall and release the highly reactive oxygenfree radicals, NO would be destroyed, rapidly oxidised and inactivated. Meanwhile, the expression of OLDL-damaged NOS would decrease and the NO synthesis would be reduced. Under certain conditions (e.g., hypercholesterolemia), NO synthesis would be significantly reduced $^{[32,33]}$, resulting in vasodilation disorders and vasospasm. Research confirmed that NO deficiency could promote the expression of LOX-1 through NF- $\kappa \mathrm{B}$, increase the cellular uptake of OLDL and promote the accumulation of macrophage lipids and the formation of foam cells ${ }^{[34,35]}$. The experimental results of immunohistochemistry showed that compared with control group, the expression of eNOS was decreased and ET-1 was increased in the model group. XMJ could induce the expression of eNOS which significantly improved the level of NO and could reduce the expression of ET-1, which all had association with the change of LOX-1. Moreover, the high-dose XMJ group showed the best effects.

In summary, XMJ could effectively reduce the lipid level of the experimental AS rabbits and might play antiAS effect through the following mechanisms, XMJ reduced the level of ICAM-1, VCAM-1, NF-кB, MMP and MDA, induced SOD level, thus improving the antiinflammatory and antioxidation capacity, reducing LDL oxidation and OLDL-mediated vascular injuries, and eventually delayed the formation of AS; XMJ increased the expression of ET and eNOS, promoted the release of NO, reduced the LOX-1 expression and blocked the damage of OLDL towards the blood vessels (fig. 5). The results of this study provided evidence for the antiinflammatory and antioxidation effects of XMJ, which were all associated with LOX-1, thus providing an experimental basis for the further development and clinical application of XMJ. However, the exact mechanism of XMJ is not entirely clear and needs further research.

\section{Acknowledgements:}

This work was supported by National Natural Science Foundation of China (No: 81800051, 
81570723, 81673423, U1704168), Natural Science Foundation of Henan Province (162300410216, 182300410332, 201810472019) and Research Project of Xinxiang Medical University (2016PN-KFKT-02, XYBSKYZZ201626, 2017CHANXUEYAN, 2017BSQDJF). This work was supported by vascular remodelling intervention and molecular targeted therapy drug development innovation team and supported by cardiovascular remodelling intervention and molecular targeting drug research and development key laboratory. Ping Song and Jian Xu are the distinguished professors of Xinxiang Medical University; Yaling Yin is a creative talent in the education department of Henan and Taihang young professional scholar of Xinxiang Medical University; Peng Li is an academic and technical leader of the Henan provincial education department and the young backbone teacher of colleges and universities in Henan.

\section{Conflict of interest:}

The authors declare that there is no conflict of interest associated with this work.

\section{REFERENCES}

1. WHO. Global Status Report on Non-communicable Diseases 2014. Geneva, Switzerland: WHO; 2014. Available from: https://www.who.int/nmh/publications/ncd-statusreport-2014/en/.

2. Arnao V, Tuttolomondo A, Daidone M, Pinto A. Lipoproteins in Atherosclerosis Process. Curr Med Chem 2019;26:1525-43.

3. Ishino S, Mukai T, Kume N, Asano D, Ogawa M, Kuge Y, et al. Lectin-like oxidized LDL receptor-1 (LOX-1) expression is associated with atherosclerotic plaque instability--analysis in hypercholesterolemic rabbits. Atherosclerosis 2007;195:4856.

4. Pirillo A, Norata GD, Catapano AL. LOX-1, OxLDL, and atherosclerosis. Mediators Inflamm 2013;2013:152786.

5. Xu S, Ogura S, Chen J, Little PJ, Moss J, Liu P. LOX-1 in atherosclerosis: biological functions and pharmacological modifiers. Cell Mol Life Sci 2013;70:2859-72.

6. Mitra S, Goyal T, Mehta JL. Oxidized, LDL, LOX-1 and atherosclerosis. Cardiovasc Drugs Ther 2011;25:419-29.

7. Chen M, Kakutani M, Minami M, Kataoka H, Kume N, Narumiya S, et al. Increased expression of lectin-like oxidized low density lipoprotein receptor-1 in initial atherosclerotic lesions of Watanabe heritable hyperlipidemic rabbits. Arterioscler Thromb Vasc Biol 2000;20:1107-15.

8. Mehta JL, Sanada N, Hu CP, Chen J, Dandapat A, Sugawara $\mathrm{F}$, et al. Deletion of LOX-1 reduces atherogenesis in LDLR knockout mice fed high cholesterol diet. Circ Res 2007;100:1634-42.

9. Balin M, Celik A, Kobat MA. The association between soluble lectin-like oxidized low-density lipoprotein receptor-1 levels and patients with isolated coronary artery ectasia. J Thromb Thrombol 2012;33:239-45.

10. Wan J, Yin Y, Sun R, Pan G, Li P, Jia Y, et al. Protective effect of the ultra-filtration extract from Xin Mai Jia on human aortic smooth muscle cell injury induced by hydrogen peroxide. Exp Ther Med 2014;7:11-6.

11. Li P, Pan GP, Jia M, Wang QQ, Guo ZG, Zhao FR, et al. Effect of Xin Mai Jia on atherosclerosis in rats. Genet Mol Res 2015;14:6018-27.

12. Zhao FR, Lu JX, Jia M, Yin YL, Qi HT, Zhu ML, et al. Effects and mechanism of Xin Mai Jia in a rabbit model of atherosclerosis. Exp Ther Med 2015;10:1627-34.

13. Lu JX, Guo C, Ou WS, Jing Y, Niu HF, Song P, et al. Citronellal prevents endothelial dysfunction and atherosclerosis in rats. J Cell Biochem 2019;120:3790-800.

14. Emini Veseli B, Perrotta P, De Meyer GRA, Roth L, Van der Donckt C, Martinet W, et al. Animal models of atherosclerosis. Eur J Pharmacol 2017;816:3-13.

15. Feng B, Yang TS, Zhang HW. Establishment of atherosclerosis models induced by feeding high-fat diet plus arterial intimal injury of the ventral aorta with balloon in rabbits. J Clin Rehabilitative Tissue Eng Res 2009;15:2911-14.

16. Fu WJ, Lei T, Yin Z, Pan JH, Chai YS, Xu XY, et al. Antiatherosclerosis and cardio-protective effects of the Angong Niuhuang Pill on a high fat and vitamin D3 induced rodent model of atherosclerosis. J Ethnopharmacol 2017;195:118-26.

17. Jin P, Cong S. LOX-1 and atherosclerotic-related diseases. Clin Chim Acta 2019;491:24-29.

18. Chen B, Wang W, Shen T, Qi R. Thioredoxin1 Downregulates Oxidized Low-Density Lipoprotein-Induced Adhesion Molecule Expression via Smad3 Protein. PloS One 2013;8:e76226.

19. Zhang HP, Zheng FL, Zhao JH, Guo DX, Chen XL. Genistein inhibits ox-LDL-induced VCAM-1, ICAM-1 and MCP-1 expression of HUVECs through heme oxygenase-1. Arch Med Res 2013;44:13-20.

20. Huo Y, Ley K. Adhesion molecules and atherogenesis. Acta Physiol Scand 2001;173:35-43.

21. Vigetti D, Rizzi M, Viola M, Karousou E, Genasetti A, Clerici $\mathrm{M}$, et al. The effects of 4-methylumbelliferone on hyaluronan synthesis, MMP2 activity, proliferation, and motility of human aortic smooth muscle cells. Glycobiology 2009;19:537-46.

22. Hakuno D, Kimura N, Yoshioka M, Mukai M, Kimura T, Okada $\mathrm{Y}$, et al. Periostin advances atherosclerotic and rheumatic cardiac valve degeneration by inducing angiogenesis and MMP production in humans and rodents. J Clin Invest 2010;120:2292-306.

23. Fiotti N, Xiong W, Giansante C. MMP-2 genetic variant and plaque features of instability. Atherosclerosis 2010;210:43-44.

24. Rudijanto A. Calcium channel blocker (diltiazem) inhibits apoptosis of vascular smooth muscle cell exposed to high glucose concentration through lectin-like oxidized low density lipoprotein receptor-1 (LOX-1) pathway. Acta Med Indones 2010;42:59-65.

25. Nagahama Y, Obama T, Usui M, Kanazawa Y, Iwamoto S, Suzuki K, et al. Oxidized low-density lipoprotein-induced periodontal inflammation is associated with the up-regulation of cyclooxygenase- 2 and microsomal prostaglandin synthase 1 in human gingival epithelial cells. Biochem Biophys Res Commun 2011;413:566-71.

26. Zhang M, Zhang Y, Zhu S, Li X, Yang Q, Bai H, et al. Genetic variants of the class A scavenger receptor gene are associated with coronary artery disease in Chinese. J Biomed Res 2012;26:418-24.

27. Casciani E, De Vincentiis C, Colaiacomo MC, Gualdi GF. 
Multi-modal imaging technologies in cardiovascular risk assessment. Ther Apher Dial 2013;17:138-49.

28. Chakraborty M, Lou C, Huan C, Kuo MS, Park TS, Cao G, et al. Myeloid cell-specific serine palmitoyltransferase subunit 2 haploinsufficiency reduces murine atherosclerosis. J Clin Invest 2013;123:1784-97.

29. Chumakova GA, Veselovskaia NG, Gritsenko OV, Kozarenko AA, Subbotin EA. Epicardial adiposity as risk factor of coronary atherosclerosis. Kardiologiia 2013;53:51-6.

30. Bao MH, Zhang YW, Zhou HH. Paeonol suppresses oxidized low-density lipoprotein induced endothelial cell apoptosis via activation of LOX-1/p38MAPK/NF- $\kappa$ B pathway. J Ethnopharmacol 2013;146:543-51.

31. Sakurai K, Cominacini L, Garbin U, Fratta Pasini A, Sasaki $\mathrm{N}$, Takuwa $\mathrm{Y}$, et al. Induction of endothelin-1 production in endothelial cells via co-operative action between CD40 and lectin-like oxidized LDL receptor (LOX-1). J Cardiovasc Pharmacol 2004;44:S173-80.
32. de Beer VJ, Merkus D, Bender SB, Tharp DL, Bowles DK, Duncker DJ, et al. Familial hypercholesterolemia impairs exercise-induced systemic vasodilation due to reduced NO bioavailability. J Appl Physiol 2013;115:1767-76.

33. Leiva A, de Medina CD, Salsoso R, Saez T, San Martin S, Abarzua F, et al. Maternal hypercholesterolemia in pregnancy associates with umbilical vein endothelial dysfunction: role of endothelial nitric oxide synthase and arginase II. Arterioscler. Thromb Vasc Biol 2013;33:2444-53.

34. Smirnova IV, Kajstura M, Sawamura T, Goligorsky MS. Asymmetric dimethylarginine upregulates LOX-1 in activated macrophages: role in foam cell formation. Am J Physiol Heart Circ Physiol 2004; 287:H782-90.

35. Mita S, Kobayashi N, Yoshida K, Nakano S, Matsuoka H. Cardioprotective mechanisms of Rho-kinase inhibition associated with eNOS and oxidative stress-LOX-1 pathway in Dahl salt-sensitive hypertensive rats. J Hypertens 2005;23:8796. 